\title{
Effector system-specific sequential modulations of congruency effects
}

\author{
Markus Janczyk $^{1} \cdot$ Hartmut Leuthold ${ }^{1}$
}

Published online: 12 June 2017

(C) Psychonomic Society, Inc. 2017

\begin{abstract}
Congruency effects in conflict tasks are reliably reduced after experiencing conflict, that is, following incongruent trials. Such sequential modulations (sometimes referred to as the Gratton effect) indicate the operation of conflict adaptation mechanisms. The influential conflict monitoring hypothesis suggested that after conflict the processing of relevant stimulus dimensions is increased. Alternatively, it was suggested that the influence of automatic response activation is suppressed following conflict. In two experiments, participants worked on the same cognitive task (Experiment 1 : Eriksen flanker; Experiment 2: Simon) with the same kind of stimulation. A cue indicated whether they should respond with the hands or the feet. When the effector system repeated from the previous trial, a sequential modulation was reliably observed. When the effector system switched, however, the sequential modulation collapsed. These results are taken to argue for the suppression of effector system-specific response activations as a consequence of experiencing conflict. Alternative interpretations in terms of task-set and/or context switches are discussed.
\end{abstract}

Keywords Congruency effect $\cdot$ Conflict adaptation · Sequential modulation $\cdot$ Inhibition $\cdot$ Effector systems

Electronic supplementary material The online version of this article (doi:10.3758/s13423-017-1311-y) contains supplementary material, which is available to authorized users.

Markus Janczyk

markus.janczyk@uni-tuebingen.de

1 Department of Psychology, Eberhard Karls University of Tübingen, Schleichstraße 4, 72076 Tübingen, Germany
Life has routinely situations where the environment affords several behavioral options that are, however, incompatible. Seeing somebody crossing the street against a red traffic light suggests that one does so also, but at the same time the red traffic light indicates one to stop. How the cognitive system deals with such conflicts has become a lively research topic in cognitive psychology over the past 2 decades. In the laboratory, conflict is usually induced with tasks where a relevant stimulus (or stimulus feature) calls for one particular response while some other simultaneously present stimulus (or stimulus feature) suggests either the same response (in congruent noconflict trials) or another response (in incongruent conflict trials). The most well-known conflict tasks are the Simon task (Simon \& Rudell, 1967), the Eriksen flanker task (Eriksen \& Eriksen, 1974), and the Stroop task (Stroop, 1935). The standard outcome is the congruency effect with shorter response times (RTs) and less errors in congruent compared to incongruent trials.

The size of the congruency effect is reliably influenced by the congruency of the preceding trial $n$ - 1 . In particular, in the Eriksen flanker task, the congruency effect is larger following congruent than following incongruent trials $n-1$ (Gratton, Coles, \& Donchin, 1992). This sequential modulation, sometimes referred to as the Gratton or congruency sequence effect (CSE), has later been reported for other conflict tasks (Simon: Praamstra, Kleine, \& Schnitzler, 1999; see also Stürmer, Leuthold, Soetens, Schröter, \& Sommer, 2002; Wühr, 2004; Stroop: Kerns et al., 2004; Notebaert, Gevers, Verbruggen, \& Liefooghe, 2006; dual-task situations: Janczyk, 2016), and also other effectors than manual responses (eye movements: Leuthold \& Schröter, 2006; pedal foot responses: Leuthold \& Schröter, 2006; vocal responses: Wühr, 2006).

The CSE suggests that experiencing conflict leads to adaptations in the way subsequently incoming information is processed within the cognitive system (for a review, see Egner, 
2007). According to the influential conflict monitoring hypothesis (Botvinick, Braver, Barch, Carter, \& Cohen, 2001), the registration of conflict (e.g., due to competing response tendencies) leads to an increased processing of the relevant stimulus (dimension), that is, the adjustments relates to processing of incoming information. In a strict interpretation then, switching the effector system with which the response is given should play no role, as long as the stimulus set and the cognitive task remain the same. Stürmer et al. (2002) suggested a slightly different account for the Simon task, namely, that the activation of response tendencies via the evolved stimulus-response ( $\mathrm{S}-\mathrm{R}$ ) associations of the automatic route is suppressed following experienced conflict, that is, following an incompatible trial $n$-1 (see also Kim, Lee, \& Cho, 2015). More specifically, Stürmer and Leuthold (2003) showed that response activation within the primary motor cortex (MI) is suppressed. In other words, here the adjustments do not (only) happen on the stimulus (S) but pertain also to the respective response (R) and/or the S-R association. Such a suppression of response activation might not be restricted to the Simon task but apply more generally when an irrelevant spatial stimulus feature (e.g., arrow direction) pre-activates a corresponding response (e.g., Eimer, 1995). In this respect, it is important to note that MI activation of hand and foot is neurofunctionally separate and hence effector system-specific, agreeing with their distinct neuroanatomic representations on the lateral (hand) versus medial (foot) MI (cf. Jentzsch \& Leuthold, 2002). Also, hand-foot compared to hand-hand response combinations produce less interference in the stopsignal paradigm (De Jong, Coles, \& Logan, 1995) and in the psychological refractory period (PRP) task (De Jong, 1993), further supporting the view that hand and foot responses are relatively independently processed. In sum, experiencing an incompatible trial leads to the suppression of the automatic route within the currently employed effector system and thereby to a diminished congruency effect when the effector system repeats. Because the automatic route of other effector systems is not affected from this suppression, changing the effector system escapes the adjustment effects following experienced conflict on an incompatible trial. Thus, and in contrast to a strict interpretation of Botvinick et al. (2001), CSEs are only expected if the effector system is unchanged in subsequent trials, but diminished CSEs are expected if the effector system switches, even if the stimulus set and the cognitive task remain the same.

Such specificity of sequential modulations is a central question in conflict research, with a focus on whether conflict induced in one task (e.g., a Simon task) has consequences for performance in a different subsequent task (e.g., a flanker task). In the majority of studies, no generalized conflict adaptations were reported (see Braem, Abrahamse, Duthoo, \& Notebaert, 2014, for a review). Preliminary evidence for a break-down of sequential modulations after switching the effectors can be taken from Experiment 1 of Akçay and
Hazeltine (2008). In the two-color condition of this experiment, participants responded to the color of a stimulus with either a left or a right finger of the left or right hand. The hand used was indicated by the hemifield in which the stimulus occurred. In both hemifields, relative left and right stimulus locations were provided (relative Simon task). When successive stimuli occurred in the same hemifield - and thus the effector hand was repeated - a clear CSE was observed. However, this was not the case when the hemifield - and thus the effector hand-switched from previous to current trial. Only after eliminating stimulus repetitions from the latter condition did a CSE occur.

We report two experiments, where the cognitive task and the stimulus set remained the same in all trials. Because of the invariant stimulus set, also the task-relevant stimulus information remained constant, which was shown to be a prerequisite for generalized conflict adaptation across different tasks (Notebaert \& Verguts, 2008). Similar to the seminal study by Gratton et al. (1992), we employed an Eriksen flanker task in Experiment 1. However, we reverted to use arrow stimuli (in contrast to arbitrary letters) to more efficiently induce an automatic response activation via the direction of the flanking arrows (cf. Eimer, 1995). Admittedly, automatic response activation is usually induced by stimulus inherent features such as their location. Thus, we complemented the flanker experiment by employing a visual, horizontal Simon task in Experiment 2. The crucial variation relates to the effector system: A cue presented at the beginning of each trial indicated whether participants had to give their response with a left/right manual key press or with a left/right pedal foot response. For repetitions of the effector system (hands vs. feet), we expected the standard CSE, that is, a smaller congruency effect following incongruent than following congruent trials. If enhanced processing of the relevant stimulus dimension (Botvinick et al., 2001) represents the mechanism of conflict adaptation, the same CSE should be observed when the effector system is switched from trial $n$-1. If, in contrast, effector system-specific S-R links are suppressed so that they cannot exert their influence in the subsequent trial, no (or a reduced) CSE should be evident when the effector system switches.

\section{Experiment 1 (Eriksen flanker task)}

\section{Method}

Participants Thirty-six students (mean age: 22.5 years, 26 female) from the University of Tübingen participated for monetary compensation or course credit. Participants were naïve regarding the hypotheses of the experiment, reported correct or corrected-to-normal vision, and signed written informed consent prior to experimentation. G*Power (Faul, Erdfelder, Lang, \& Buchner, 2007) calculated a required sample size of $n$ 
$=34$ to detect a medium-sized effect of $d=0.5$ with a power of $1-\beta=.8$.

Apparatus and stimuli The experimental procedure was controlled by a standard PC connected to a 17-in. CRT monitor. Manual responses were given via two custombuilt response keys located on a table to the left and right of the participant. Foot responses were given via two footswitches placed under the table in a position comfortable for the participant. Stimuli (targets) were single left/right-pointing arrows $(</>)$, and double arrows $(<</>>)$ were used as flankers. The target was presented in the screen center, two flankers of the same kind were located to the target's left and right. The letters $H$ and $F$ were used as cues indicating whether a hand or a foot response, respectively, is required in the current trial.

Task and procedure The participants' task was to give a left/right manual hand response or a left/right foot response (as signaled by the cue letter), according to the direction of the (central) target arrow.

Each trial started with the presentation of the cue letter (500 ms), followed by a blank screen $(500 \mathrm{~ms})$. Then the flankers appeared, and $100 \mathrm{~ms}$ later the central target, staying on screen until a response was given or the trial was canceled after 2,000 ms without a response. In case of errors (wrong response location, wrong effector, no response given), error feedback was given for 1,000 ms. The next trial started after a blank inter trial interval of $1,000 \mathrm{~ms}$.

Participants started with 10 randomly drawn familiarization trials that were followed by nine blocks of 64 trials each, resulting from eight repetitions of the combinations of 2 effector systems (hands vs. feet) $\times 2$ target directions (left vs. right) $\times 2$ flanker directions (left vs. right) that were randomly presented. Instructions were given in written form on screen prior to the experiment. An experimental session lasted about 45 minutes.

Design and analyses A trial was congruent if target and flankers pointed to the same direction; otherwise it was incongruent. Three variables of interest were coded: (1) the congruency of the current trial $n$, (2) the congruency of the previous trial $n$-1, and (3) whether the effector system repeated or switched from trial $n-1$.

The first experimental block (practice) and the first trial of each other block were excluded from analyses. For RT analyses, only correct trials that followed a correct trial $n-1$ were considered. Also, outliers were excluded if an RT deviated by more than three standard deviations from the mean (calculated for each participant and design cell separately). Error analyses were based on trials involving a wrong response location following a correct trial $n-1$.
Mean correct RTs and percentages error (PE) were submitted to $2 \times 2 \times 2$ analyses of variance (ANOVAs) with repeated measures on all three variables of interest.

\section{Results and discussion}

Mean correct RTs (2.32\% outliers) are visualized in Fig. 1 (see also Table 1). Overall, responses were slower in incongruent compared with congruent trials, $F(1,35)=316.12, p<.001$, $\eta_{\mathrm{p}}{ }^{2}=.90$, but this congruency effect was modulated by trial $n$ 1 congruency. In particular, its size became smaller following incongruent than congruent trials $n-1, F(1,35)=13.61$, $p<.001, \eta_{\mathrm{p}}{ }^{2}=.28$, indicating a CSE. Neither effector system switch, $F(1,35)=0.45, p=.507, \eta_{\mathrm{p}}{ }^{2}=.01$, nor congruency of trial $n-1$ exerted a main effect, $F(1,35)=0.37, p=.548, \eta_{\mathrm{p}}{ }^{2}=.01$. Also, the interactions between effector system switch and congruency in trial $n, F(1,35)=1.76, p=.193, \eta_{\mathrm{p}}{ }^{2}=.05$, and of effector system switch and congruency in trial $n-1, F(1,35)=$ $0.11, p=.748, \eta_{\mathrm{p}}{ }^{2}<.01$, were not significant. However, the three-way interaction was significant, $F(1,35)=6.25, p=.017$, $\eta_{\mathrm{p}}{ }^{2}=.15$, pointing to different interactions of congruency in trial $n$ and trial $n-1$ for effector system repetitions and switches. Thus, both conditions were analyzed separately with $2 \times 2$ ANOVAs with congruency in trials $n$ and $n-1$ as repeated measures.

For effector system repetitions, the main effect of trial $n$ congruency was significant, $F(1,35)=250.90, p<.001, \eta_{\mathrm{p}}{ }^{2}=.88$, but that of trial $n-1$ was not, $F(1,35)=0.48, p=.493, \eta_{\mathrm{p}}{ }^{2}=.01$. Critically, the interaction indicating the CSE was significant, $F(1,35)=19.25, p<.001, \eta_{\mathrm{p}}{ }^{2}=.35$. For effector system switches, the main effect of trial $n$ congruency was also significant, $F(1,35)=278.08, p<.001, \eta_{\mathrm{p}}{ }^{2}=.80$, but neither that of trial $n-1, F(1,35)=0.01, p=.933, \eta_{\mathrm{p}}{ }^{2}<.01$, nor the interaction were significant, $F(1,35)=0.73, p=.400, \eta_{\mathrm{p}}{ }^{2}=.02$.

Mean PEs are summarized in Table 1 (for full ANOVA results, see the supplementary online material). Important for the present purpose, the interaction between trial $n$ and trial $n$ 1 congruency was significant, $F(1,35)=31.37, p<.001, \eta_{\mathrm{p}}{ }^{2}=$ .47 , but this interaction was not modulated by effector system switches, $F(1,35)=1.07, p=.308, \eta_{\mathrm{p}}{ }^{2}=.03$.

When considering RTs, we observed a pattern compatible with the idea that the influence of effector systemspecific S-R associations is reduced following incongruent trials. With effector system repetitions, a sequential modulation was observed with a smaller congruency effect following incongruent than following congruent trials $n-1$. When the effector system switched, in contrast, no such sequential modulation was observed. However, a similar sequential modulation for effector system repetitions and switches was present in error data, thus leaving us with a slightly unclear pattern. Also, while the flanker effect itself was large, the sequential modulation was relatively small in comparison. Before drawing further conclusions, we present Experiment 2. 


\section{Effector system repetition}

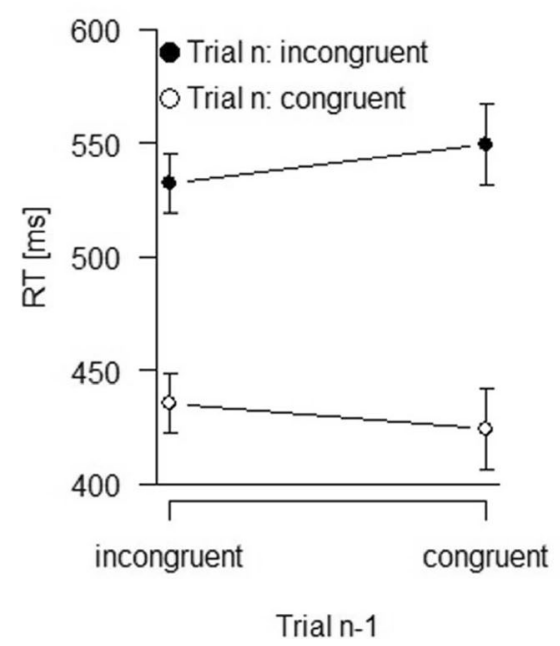

Fig. 1 Mean correct RTs from Experiment 1 as a function of effector system repetition (left panel) versus effector system switch (right panel) and congruency in trial $n$ and in trial $n-1$. Error bars are $95 \%$ confidence

\section{Experiment 2 (Simon task)}

\section{Method}

Participants Thirty-six fresh students (mean age: 23.8 years, 30 female) from the University of Tübingen participated for monetary compensation or course credit, fulfilling the same criteria as in Experiment 1.

Apparatus and stimuli The same apparatus, response keys, foot pedals, and effector cues as in Experiment 1 were used. Stimuli were red and green filled circles, presented to the left or the right of the screen center.

Task and procedure The participants' task was to give a left/right manual hand response or a left/right foot response (as signaled by the cue letter), according to the color of the circle.

\section{Effector system switch}

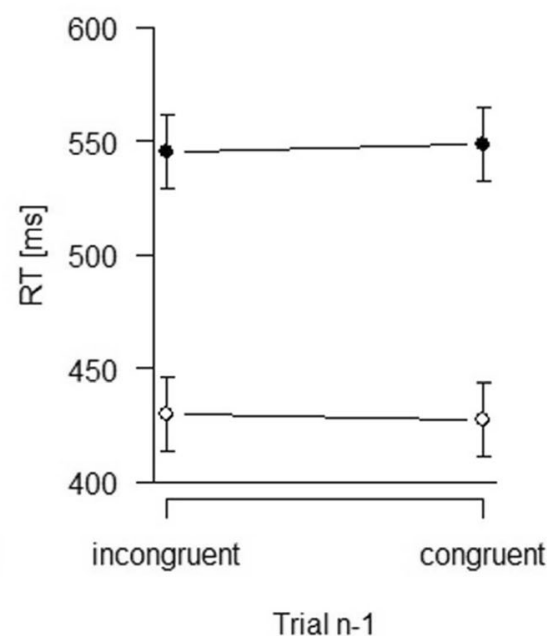

intervals for the difference between congruent and incongruent trials $n$, separately for each trial $n-1$ congruency and effector system repetition/ switch (see Pfister \& Janczyk, 2013)

Each trial started with the presentation of the cue letter $(500 \mathrm{~ms})$, followed by a blank screen $(500 \mathrm{~ms})$, and the onset of the lateralized target circle, staying on screen until a response was given or the trial was canceled after 2,000 ms without a response. In case of errors (wrong response location, wrong effector, no response given), error feedback was given for $1,000 \mathrm{~ms}$. The next trial started after a blank inter trial interval of $1,000 \mathrm{~ms}$.

Participants started with 10 randomly drawn familiarization trials that were followed by nine blocks of 64 trials each, resulting from eight repetitions of the combinations of 2 effector systems (hands vs. feet) $\times 2$ target colors (red vs green) $\times 2$ target locations (left vs. right) that were randomly presented. Instructions were given in written form on-screen prior to the experiment. An experimental session lasted about 45 minutes.

Design and analyses A trial was congruent if target location and response location matched. In all other aspects, design and analyses were as described for Experiment 1.

Table 1 Mean correct RTs and percentages error from Experiment 1 as a function of effector system repetition/switch and congruency in trial $n$ and in trial $n-1$

\begin{tabular}{|c|c|c|c|c|c|c|c|c|}
\hline & \multicolumn{4}{|c|}{ Response times [ms] } & \multicolumn{4}{|c|}{ Percentage error } \\
\hline & \multicolumn{2}{|c|}{ Effector system repetition } & \multicolumn{2}{|c|}{ Effector system switch } & \multicolumn{2}{|c|}{ Effector system repetition } & \multicolumn{2}{|c|}{ Effector system switch } \\
\hline & \multicolumn{2}{|l|}{ Trial $n-1$} & \multicolumn{2}{|c|}{ Trial $n-1$} & \multicolumn{2}{|c|}{ Trial $n-1$} & \multicolumn{2}{|c|}{ Trial $n-1$} \\
\hline Trial $n$ & Incong & Cong & Incong & Cong & Incong & Cong & Incong & Cong \\
\hline Incong & 533 & 549 & 545 & 549 & 3.8 & 7.1 & 3.1 & 6.0 \\
\hline Cong & 436 & 424 & 430 & 427 & 1.2 & 0.6 & 0.8 & 1.1 \\
\hline
\end{tabular}

Note. Incong = incongruent; Cong $=$ congruent 


\section{Effector system repetition}

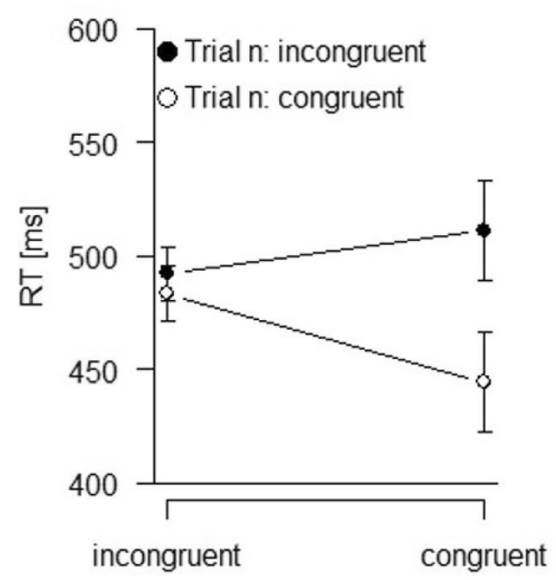

Trial $n-1$

Fig. 2 Mean correct RTs from Experiment 2 as a function of effector system repetition (left panel) versus effector system switch (right panel) and congruency in trial $n$ and in trial $n-1$. Error bars are $95 \%$ confidence

\section{Results and discussion}

Mean correct RTs (2.04\% outliers) are visualized in Fig. 2 (see also Table 2). Overall, responses were slower in incongruent than congruent trials, $F(1,35)=39.11, p<.001, \eta_{\mathrm{p}}{ }^{2}=.53$, and with effector system switches compared to repetitions, $F(1,35)=$ $50.76, p<.001, \eta_{\mathrm{p}}^{2}=.59$. Congruency in trial $n-1$ had no main effect, $F(1,35)=2.47, p=.125, \eta_{\mathrm{p}}{ }^{2}=.07$, but it interacted with congruency in trial $n$, indicating the CSE, $F(1,35)=18.31, p<$ $.001, \eta_{\mathrm{p}}{ }^{2}=.34$. The interactions between effector system switch and congruency in trial $n, F(1,35)<.001, p=.953, \eta_{\mathrm{p}}^{2}<.01$, and of effector system switch and congruency in trial $n-1, F(1,35)=$ $0.51, p=.479, \eta_{\mathrm{p}}^{2}=.01$, were not significant, whereas the threeway interaction was, $F(1,35)=6.57, p=.015, \eta_{\mathrm{p}}^{2}=.15$.

For effector system repetitions, the main effect of trial $n$ congruency was significant, $F(1,35)=34.29, p<.001, \eta_{\mathrm{p}}{ }^{2}=$ .49 , and that of trial $n-1$ just missed significance, $F(1,35)=$ $3.75, p=.061, \eta_{\mathrm{p}}{ }^{2}=.10$. Critically, the CSE indicating interaction was significant, $F(1,35)=27.53, p<.001, \eta_{\mathrm{p}}{ }^{2}=.44$. For effector system switches, only the main effect of trial $n$

\section{Effector system switch}

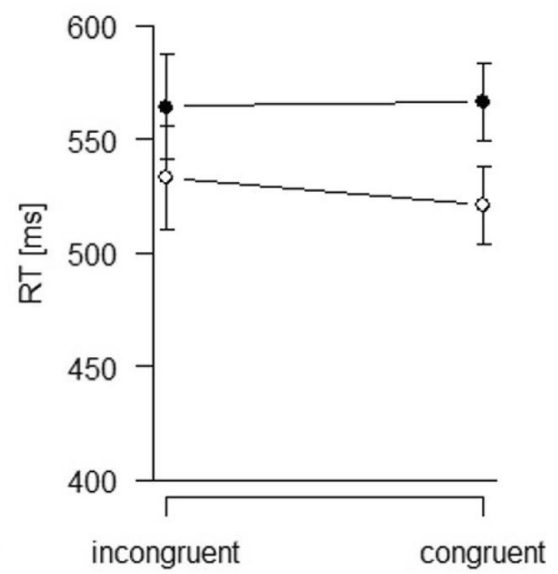

Trial $n-1$

intervals for the difference between congruent and incongruent trials $n$, separately for each trial $n-1$ congruency and effector system repetition/ switch (see Pfister \& Janczyk, 2013)

congruency was significant, $F(1,35)=24.13, p<.001, \eta_{\mathrm{p}}{ }^{2}=$ .41. Neither the main effect of trial $n-1$ congruency, $F(1,35)=$ $0.62, p=.437, \eta_{\mathrm{p}}^{2}=.02$, nor the interaction were significant, $F(1,35)=1.21, p=.279, \eta_{\mathrm{p}}{ }^{2}=.03$.

Mean PEs are summarized in Table 2 (for the full ANOVA results, see the supplementary online material). Crucially, the interaction between trial $n$ and trial $n$-1 congruency was significant, $F(1,35)=10.57, p=.003, \eta_{\mathrm{p}}^{2}=.15$, but this interaction was modulated by effector system switches, $F(1,35)=$ $7.53, p=.010, \eta_{\mathrm{p}}{ }^{2}=.18$ : While trial $n$ and trial $n-1$ congruency interacted for effector system repetitions, $F(1,35)=15.40$, $p<.001, \eta_{\mathrm{p}}^{2}=.31$, this was not the case for effector system switches, $F(1,35)=0.03, p=.860, \eta_{\mathrm{p}}^{2}=.01$.

By and large, results from this experiment replicate those from Experiment 1. With effector system repetitions, a clear CSE occurred. With effector system switches, however, no CSE was observed in RTs or for error data. Thus, Experiment 2 clearly suggests that changing the effector system eliminates any conflict adaptations resulting from the previous trial $n-1$.

Table 2 Mean correct RTs and percentages error from Experiment 2 as a function of effector system repetition/switch and congruency in trial $n$ and in trial $n-1$

\begin{tabular}{|c|c|c|c|c|c|c|c|c|}
\hline & \multicolumn{4}{|c|}{ Response times [ms] } & \multicolumn{4}{|c|}{ Percentage error } \\
\hline & \multicolumn{2}{|c|}{ Effector system repetition } & \multicolumn{2}{|c|}{ Effector system switch } & \multicolumn{2}{|c|}{ Effector repetition system } & \multicolumn{2}{|c|}{ Effector switch system } \\
\hline & Trial $n-1$ & & Trial $n-1$ & & Trial $n-1$ & & Trial $n-$ & \\
\hline Trial $n$ & Incong & Cong & Incong & Cong & Incong & Cong & Incong & Cong \\
\hline Incong & 492 & 511 & 564 & 566 & 2.2 & 5.3 & 8.3 & 7.0 \\
\hline Cong & 483 & 444 & 533 & 521 & 2.8 & 0.8 & 4.6 & 3.1 \\
\hline
\end{tabular}

Note. Incong = incongruent; Cong = congruent 


\section{General discussion}

In two experiments, we assessed the CSE when the kind of stimulation and the cognitive task were the same in all trials (Experiment 1: Eriksen flanker task; Experiment 2: Simon task), but participants responded either with their hands or their feet according to a cue presented before the stimulation.

\section{Summary of results}

When the effector system is repeated, a CSE with smaller congruency effects following incongruent than following congruent trials $n$-1 was observed (e.g., Gratton et al., 1992). In contrast, when the effector system switched from the previous trial $n-1$ to the current trial $n$, no such CSE was observed. ${ }^{1}$ Inasmuch the CSE is taken as an index of conflict adaptation (e.g., Botvinick et al., 2001), this latter result indicates that conflict adaptation depends on the involved effector system. Thus, conflict adaptation cannot only result in increased/decreased processing of relevant stimulus features because those were exactly the same in effector system switch trials in our experiments. The result is compatible, however, with a suppression of automatic and effector system-specific response activation in MI (Jentzsch \& Leuthold, 2002; Stürmer \& Leuthold, 2003).

The result is also in line with a study by Braem, Verguts, and Notebaert (2011). These authors employed a horizontal and a vertical Simon task with one task requiring manual hand responses and the other foot responses. A standard CSE was only observed when the effector system repeated. When it switched, in contrast, even a reversed CSE was observed. Critically, while the task-relevant (and task-irrelevant) stimulus information was the same in both task versions, the different effector systems were effectively assigned to different stimuli (Braem et al., 2011, p. 1665). Thus, while in line with Braem et al.'s results, our study goes beyond theirs by having participants work on the same tasks and the same kind of stimulation with only the effector system being varied.

Our study further complements recent work by Kreutzfeldt, Stephan, Willmes, and Koch (2016), who held the effector system constant across trials (manual key presses) but varied (and cued) the relevant modality of a bimodal stimulus (auditory-visual). Similar to our results, a sequential modulation was only observed when the relevant stimulus modality repeated from the previous trial $n-1$ but not when it switched. Together, these results point to very specific conflict

\footnotetext{
${ }^{1}$ To be precise here, a CSE was observed in Experiment 1 in error data. However, this finding was not replicated in Experiment 2, and a CSE was never observed for RTs. We thus hesitate interpreting this single exceptional finding. However, a (small) contribution of enhanced processing of relevant stimulus features can perhaps be expected in tasks with overlapping stimulus dimensions, such as the flanker task used in Experiment 1 (see Egner, 2008). In line with this, a recent study by Soutschek, Müller, and Schubert (2013) reported different effects of accessory stimuli on conflict adaptation in Stroop and Simon tasks.
}

adaptation mechanisms that affect specific stimulus and response modalities and do not necessarily generalize to other input and output modalities.

\section{Alternative interpretations}

Generalized conflict adaptation was suggested to depend on the structure of employed task sets (Akçay \& Hazeltine, 2008; Hazeltine, Lightman, Schwarb, \& Schumacher, 2011). One may argue that due to the different effector systems our participants actually represented two task sets, and an effector system switch thus implied a task set switch. This possibility is hard to rule out, but at least the cognitive task and the stimulation were the same in all trials in our experiments.

It was also argued that CSEs may result from feature integration (slower partial repetitions vs. faster full repetitions/alternations). In case of effector system switch trials, however, essentially the full repetitions in congruent-congruent or incongruentincongruent sequences are eliminated, which should still be faster than the other sequences. Thus, this account predicts the same pattern for effector system repetitions and switches (see also Kreutzfeldt et al., 2016, for a more detailed discussion).

In a broader way, Kreutzfeldt et al. (2016; see also Abrahamse, Braem, Notebaert, \& Verguts, 2016) suggested that various instances of non-generalized conflict adaptation should be interpreted as indicating switches of "contexts," which should "disrupt the continuity of cognitive processing across episodes" (p. 1467). In this sense, one could argue that the cue signaling the appropriate effector system also sets up one of two contexts and thus leads to a disruption of sequential modulations. Frankly, we cannot exclude such interpretations, but it appears hard to conceive an experiment where changes in any feature cannot be reinterpreted as a change of context.

\section{Conclusions}

In two experiments we observed that the CSE and thus conflict adaptation collapses when the effector system changes from the previous trial $n-1$ to the current trial $n$. We suggest that conflict adaptation involves the suppression of effector system-specific automatic response activation in MI.

Acknowledgements Work of M.J. is supported by the Institutional Strategy of the University of Tübingen (Deutsche Forschungsgemeinschaft [German Research Foundation], ZUK 63). We thank Magali Kreutzfeldt for helpful comments on a previous version of this manuscript.

\section{References}

Abrahamse, E., Braem, S., Notebaert, W., \& Verguts, T. (2016). Grounding cognitive control in associative learning. Psychological Bulletin, 142, 693-728. 
Akçay, Ç., \& Hazeltine, E. (2008). Conflict adaptation depends on task structure. Journal of Experimental Psychology: Human Perception and Performance, 34, 958-973.

Botvinick, M. M., Braver, T. S., Barch, D. M., Carter, C. S., \& Cohen, J. D. (2001). Conflict monitoring and cognitive control. Psychological Review, 108, 624-652.

Braem, S., Abrahamse, E. L., Duthoo, W., \& Notebaert, W. (2014). What determines the specificity of conflict adaptation? A review, critical analysis, and proposed synthesis. Frontiers in Psychology, 5, 1134. doi:10.3389/fpsyg.2014.01134

Braem, S., Verguts, T., \& Notebaert, W. (2011). Conflict adaptation by means of associative learning. Journal of Experimental Psychology: Human Perception and Performance, 37, 1662-1666.

De Jong, R. (1993). Multiple bottlenecks in overlapping task performance. Journal of Experimental Psychology: Human Perception and Performance, 19, 965-980.

De Jong, R., Coles, M. G. H., \& Logan, G. D. (1995). Strategies and mechanisms in nonselective and selective inhibitory motor control. Journal of Experimental Psychology: Human Perception and Performance, 21, 498-511.

Egner, T. (2007). Congruency sequence effects and cognitive control. Cognitive, Affective, \& Behavioral Neuroscience, 7, 380-390.

Egner, T. (2008). Multiple conflict-driven control mechanisms in the human brain. Trends in Cognitive Sciences, 12, 374-380.

Eimer, M. (1995). Stimulus-response compatibility and automatic response activation: Evidence form electrophysiological studies. Journal of Experimental Psychology: Human Perception and Performance, 21, 837-854.

Eriksen, B. A., \& Eriksen, C. W. (1974). Effects of noise letters upon the identification of a target letter in a nonsearch task. Perception \& Psychophysics, 16, 143-149.

Faul, F., Erdfelder, E., Lang, A.-G., \& Buchner, A. (2007). G*Power 3: A flexible statistical power analysis program for the social, behavioral, and biomedical sciences. Behavior Research Methods, 39, 175-191.

Gratton, G., Coles, M. G., \& Donchin, E. (1992). Optimizing the use of information: Strategic control of activation of responses. Journal of Experimental Psychology: General, 121, 480-506.

Hazeltine, E., Lightman, E., Schwarb, H., \& Schumacher, E. H. (2011). The boundaries of sequential modulations: Evidence for set-level control. Journal of Experimental Psychology: Human Perception and Performance, 37, 1898-1914.

Janczyk, M. (2016). Sequential modulation of backward crosstalk and task-shielding in dual-tasking. Journal of Experimental Psychology: Human Perception and Performance, 42, 631-647.

Jentzsch, I., \& Leuthold, H. (2002). Advance movement preparation of eye, foot, and hand: A comparative study using movement-related brain potentials. Cognitive Brain Research, 14, 201-217.
Kerns, J. G., Cohen, J. D., MacDonald, A. W., Cho, R. Y., Stenger, V. A., \& Carter, C. S. (2004). Anterior cingulate conflict monitoring and adjustments in control. Science, 303, 1023-1026.

Kim, S., Lee, S. H., \& Cho, Y. S. (2015). Control processes through the suppression of the automatic response activation triggered by taskirrelevant information in the Simon-type tasks. Acta Psychologica, 162, 51-61.

Kreutzfeldt, M., Stephan, D. N., Willmes, K., \& Koch, I. (2016). Shifts in target modality cause attentional reset: Evidence from sequential modulation of crossmodal congruency effects. Psychonomic Bulletin \& Review, 23, 1466-1473.

Leuthold, H., \& Schröter, H. (2006). Electrophysiological evidence for response priming and conflict regulation in the auditory Simon task. Brain Research, 1097, 167-180.

Notebaert, W., Gevers, W., Verbruggen, F., \& Liefooghe, B. (2006). Topdown and bottom-up sequential modulations of congruency effects. Psychonomic Bulletin \& Review, 13, 112-117.

Notebaert, W., \& Verguts, T. (2008). Cognitive control acts locally. Cognition, 106, 1071-1080.

Pfister, R., \& Janczyk, M. (2013). Confidence intervals for two sample means: Calculation, interpretation, and a few simple rules. Advances in Cognitive Psychology, 9, 74-80.

Praamstra, P., Kleine, B. U., \& Schnitzler, A. (1999). Magnetic stimulation of the dorsal premotor cortex modulates the Simon effect. Neuroreport, 10, 3671-3674.

Simon, J. R., \& Rudell, A. P. (1967). Auditory S-R compatibility: The effect of an irrelevant cue on information processing. Journal of Applied Psychology, 51, 300-304.

Soutschek, A., Müller, H. J., \& Schubert, T. (2013). Conflict-specific effects of accessory stimuli on cognitive control in the Stroop task and the Simon task. Experimental Psychology, 60, 140-147.

Stroop, J. R. (1935). Studies of interference in serial verbal reactions. Journal of Experimental Psychology, 18, 643-662.

Stürmer, B., \& Leuthold, H. (2003). Control over response priming in visuomotor processing: A lateralized event-related potential study. Experimental Brain Research, 153, 35-44.

Stürmer, B., Leuthold, H., Soetens, E., Schröter, H., \& Sommer, W. (2002). Control over location-based response activation in the Simon task: Behavioral and electrophysiological evidence. Journal of Experimental Psychology: Human Perception and Performance, $28,1345-1363$.

Wühr, P. (2004). Sequential modulations of logical-recoding operations in the Simon task. Experimental Psychology, 51, 98-108.

Wühr, P. (2006). The Simon effect in vocal responses. Acta Psychologica, $121,210-226$. 